Algebraic 85 Geometric Topology

Volume 3 (2003) 1257-1273

Published: 20 December 2003

ATG

\title{
On a Hopf operad containing the Poisson operad
}

\author{
FRÉdÉRIC CHAPOTON
}

\begin{abstract}
A new Hopf operad Ram is introduced, which contains both the well-known Poisson operad and the Bessel operad introduced previously by the author. Besides, a structure of cooperad R is introduced on a collection of algebras given by generators and relations which have some similarity with the Arnold relations for the cohomology of the type $A$ hyperplane arrangement. A map from the operad Ram to the dual operad of $\mathrm{R}$ is defined which we conjecture to be a isomorphism.
\end{abstract}

AMS Classification 18D50; 16W30

Keywords Hopf operad, coalgebra, chain complex

\section{Introduction}

The theory of operads has roots in algebraic topology. One well-known way to build algebraic operads is to start from an operad of topological spaces and apply the homology functor. A famous example, due to Cohen $[4,5]$, is given by the little discs operad whose homology is the Gerstenhaber operad. The operads defined in this way inherit more structure from the diagonal of topological spaces: they are in fact Hopf operads. This phenomenon is similar to the existence of a bialgebra structure on the homology of a topological monoid.

This article introduces two algebraic objects. The first one is a Hopf operad called the Ramanujan operad and denoted by Ram, which contains both the well-known Poisson operad and the Bessel operad introduced in [2]. The second one is a Hopf cooperad $\mathrm{R}$, which means that the space $\mathrm{R}(I)$ associated to a finite set $I$ is an associative algebra and the cocomposition maps are morphisms of algebras.

The operad Ram is conjectured to be isomorphic to the linear dual operad $\mathrm{R}^{*}$ of the cooperad R. A morphism of operad from Ram to $R^{*}$ is defined, which should give the desired isomorphism. 
One motivation for these constructions is an analogy with the case of the Gerstenhaber operad. The dual algebras of the coalgebras underlying the Gerstenhaber operad can be presented by generators and relations, by a theorem of Arnold on the cohomology of the complexified hyperplane arrangements of type $A$. From this, one can reach a simple description of the dual cooperad of the Gerstenhaber operad. This alternative dual description of the Gerstenhaber operad is sketched at the end of the paper.

There seems to be some kind of similar relation between the cooperad R and some differential forms on the complexified hyperplane arrangements of type $A$. This relation was already proposed for the Bessel suboperad in [2]. The algebras underlying $\mathrm{R}$ are given by generators and relations which have some resemblance with the Arnold relations and seem to contain the relations satisfied by some simple differential forms.

After some preliminary material on operads in the first section, the Ramanujan operad is defined in the second section by a distributive law between the commutative operad and an operad mixing the Lie operad and the suspended Griess operad. This name has been chosen because the dimensions are supposed to be given by the so-called Ramanujan polynomials [3].

In the next section, the cooperad $\mathrm{R}$ is defined on a collection of algebras given by generators and relations. The cocomposition is motivated by the analogy with the case of the Gerstenhaber operad. Then a morphism from Ram to $\mathrm{R}^{*}$ is defined. Some algebras of differential forms are introduced, which should be related to R. Last, a construction is sketched for the Gerstenhaber operad, which motivated the formula for cocomposition in $\mathrm{R}$.

\section{Operads as functors}

Because our language for operads differs in aspect from the most frequently used setups, this section gathers conveniently some conventions and definitions.

An operad $\mathcal{P}$ is a functor from the category of finite sets and bijections to some monoidal category (sets or vector spaces for example) together with some extra structure given by composition maps. Finite sets will be denoted by capital letters $I, J, K$ and so on. Elements of finite sets will be denoted by letters $i, j, k$ and so on. In some sense, $i, j, k$ can be considered as abstract variables when they are used to denote elements of an arbitrary finite set. The symbols $\star$ and \# are used as place-holders for composition maps. 
The composition map $o_{\star}$ is defined for any two finite sets $I$ and $J$ as a map from $\mathcal{P}(I \sqcup\{\star\}) \otimes \mathcal{P}(J)$ to $\mathcal{P}(I \sqcup J)$. These composition maps have to satisfy some natural axioms. Other symbols such as \# are used instead of $\star$ when iterated compositions appear.

A presentation by generators and relations of an operad is given as follows: some generators labelled by their inputs, with some specific symmetry properties with respect to the symmetric group on these inputs, and some relations involving compositions of these generators. Consider for example the Lie operad. The generators are $L_{i, j}$ on any set $\{i, j\}$, which stand for the Lie bracket. The generator $L_{i, j}$ is antisymmetric under the exchange of $i$ and $j$. The relations are the Jacobi identities (see (1) below) on any set $\{i, j, k\}$, involving generators on various subsets of cardinality two of $\{i, j, k, \star\}$.

\section{The Ramanujan operad}

In this section, a Hopf operad Ram is defined by a distributive law. This is similar to the usual definition of the Gerstenhaber operad by a distributive law between the commutative operad and the suspended Lie operad.

\subsection{The LieGriess operad}

The ground field is $\mathbb{C}$. The ambient category is the monoidal category of $(\mathbb{Z}, \mathbb{Z})$ bigraded vector spaces endowed with two differentials of respective degree $(1,0)$ and $(-1,0)$. The Koszul sign rules for the symmetry isomorphisms of the tensor product apply only with respect to the first degree. The second degree does not play any role with respect to signs in the formulas.

One can remark that the second degree coincide in the objects considered here with the eigenvalue of the Laplacian associated to the pair of opposite differentials.

In this section, an operad LieGriess is defined which contains the operads Lie and $\Sigma$ Griess defining Lie algebras and suspended commutative non-associative algebras.

The operad LieGriess is generated by the Lie generator $L_{i, j}$ antisymmetric of degree $(0,1)$ and the $\Sigma$ Griess generator $\Omega_{i, j}$ antisymmetric of degree $(1,1)$ modulo the following relations. 
First, the Jacobi identity defining the Lie operad:

$$
\sum_{c y c l} L_{i, \star} \circ_{\star} L_{j, k}=0
$$

where the summation is over cyclic permutations of $i, j, k$.

Second, a mixed relation between the Lie generator and the $\Sigma$ Griess generator:

$$
\sum_{c y c l}\left(\Omega_{i, \star} \circ_{\star} L_{j, k}+L_{i, \star} \circ_{\star} \Omega_{j, k}\right)=0 .
$$

Note that the $\Sigma$ Griess operad is free on its generator, so there is no relation involving only $\Omega$.

\subsection{Distributive law}

For the notion of distributive law between operads, see [8].

First recall the Com operad, which is generated by $E_{i, j}$ symmetric of degree $(0,0)$ modulo the relation of associativity:

$$
E_{i, \star} \circ_{\star} E_{j, k}=E_{j, \star} \circ_{\star} E_{k, i}
$$

Then consider the following relations:

$$
\begin{aligned}
L_{i, \star} \circ_{\star} & E_{j, k}=E_{j, \star} \circ_{\star} L_{i, k}+E_{k, \star} \circ_{\star} L_{i, j}, \\
\Omega_{i, \star} \circ_{\star} E_{j, k} & =E_{j, \star} \circ_{\star} \Omega_{i, k}+E_{k, \star} \circ_{\star} \Omega_{i, j} .
\end{aligned}
$$

Proposition 1 The relations (4) and (5) define a distributive law from LieGriess $\circ$ Com to Com $\circ$ LieGriess. The resulting operad is called Ram.

Proof The relation (4), which is the Leibniz relation, is already known to define a distributive law from Lie $\circ$ Com to Com $\circ$ Lie. The resulting operad is the Poisson operad.

The relation (5) is also known to be a distributive law from $\Sigma$ Griess $\circ$ Com to Com $\circ \Sigma$ Griess which defines the Bessel operad, see [2].

So there remains only one condition to check, which comes from relation (2). One has to check that

$$
\begin{aligned}
\left(\Omega_{i, \star} \circ_{\star} L_{j, \#}+\Omega_{j, \star} \circ_{\star} L_{\#, i}+\Omega_{\#, \star} \circ_{\star} L_{i, j}+\right. \\
\left.L_{i, \star} \circ_{\star} \Omega_{j, \#}+L_{j, \star} \circ_{\star} \Omega_{\#, i}+L_{\#, \star} \circ_{\star} \Omega_{i, j}\right) \circ_{\#} E_{k, \ell},
\end{aligned}
$$

once rewritten using the distributive laws, reduces to zero modulo the relations. 
The result of rewriting is

$$
\begin{aligned}
& \Omega_{i, \star} \circ_{\star}\left(E_{\ell, \#} \circ_{\#} L_{j, k}+E_{k, \#} \circ_{\#} L_{j, \ell}\right)-\Omega_{j, \star} \circ_{\star}\left(E_{\ell, \#} \circ_{\#} L_{i, k}+E_{k, \#} \circ_{\#} L_{i, \ell}\right) \\
& +L_{i, \star} \circ_{\star}\left(E_{\ell, \#} \circ_{\#} \Omega_{j, k}+E_{k, \#} \circ_{\#} \Omega_{j, \ell}\right)-L_{j, \star} \circ_{\star}\left(E_{\ell, \#} \circ_{\#} \Omega_{i, k}+E_{k, \#} \circ_{\#} \Omega_{i, \ell}\right) \\
- & \left(E_{\ell, \#} \circ_{\#} \Omega_{\star, k}+E_{k, \#} \circ_{\#} \Omega_{\star, \ell}\right) \circ_{\star} L_{i, j}-\left(E_{\ell, \#} \circ_{\#} L_{\star, k}+E_{k, \#} \circ_{\#} L_{\star, \ell} \circ_{\star} \Omega_{i, j} .\right.
\end{aligned}
$$

This becomes, after a second application of the distributive laws,

$$
\begin{aligned}
& \left(E_{\#, \star} \circ_{\star} \Omega_{i, \ell}+E_{\ell, \star} \circ_{\star} \Omega_{i, \#}\right) \circ_{\#} L_{j, k}+\left(E_{\#, \star} \circ_{\star} \Omega_{i, k}+E_{k, \star} \circ_{\star} \Omega_{i, \#}\right) \circ_{\#} L_{j, \ell} \\
& -\left(E_{\#, \star} \circ_{\star} \Omega_{j, \ell}+E_{\ell, \star} \circ_{\star} \Omega_{j, \#}\right) \circ_{\#} L_{i, k}-\left(E_{\#, \star} \circ_{\star} \Omega_{j, k}+E_{k, \star} \circ_{\star} \Omega_{j, \#}\right) \circ_{\#} L_{i, \ell} \\
& +\left(E_{\#, \star} \circ_{\star} L_{i, \ell}+E_{\ell, \star} \circ_{\star} L_{i, \#}\right) \circ_{\#} \Omega_{j, k}+\left(E_{\#, \star} \circ_{\star} L_{i, k}+E_{k, \star} \circ_{\star} L_{i, \#}\right) \circ_{\#} \Omega_{j, \ell} \\
& -\left(E_{\#, \star} \circ_{\star} L_{j, \ell}+E_{\ell, \star} \circ_{\star} L_{j, \#}\right) \circ_{\#} \Omega_{i, k}-\left(E_{\#, \star} \circ_{\star} L_{j, k}+E_{k, \star} \circ_{\star} L_{j, \#}\right) \circ_{\#} \Omega_{i, \ell} \\
& -E_{\ell, \#} \circ_{\#}\left(\Omega_{\star, k} \circ_{\star} L_{i, j}+L_{\star, k} \circ_{\star} \Omega_{i, j}\right)-E_{k, \#} \circ_{\#}\left(\Omega_{\star, \ell} \circ_{\star} L_{i, j}+L_{\star, \ell} \circ_{\star} \Omega_{i, j}\right) .
\end{aligned}
$$

Now all 8 terms starting with $E_{\#, \star}$ annihilates pairwise and one can separate in what remains terms starting with $E_{\ell, \#}$ and with $E_{k, \#}$. Each of these separate sums is zero modulo relation (2).

The bigraded dimensions of the Ram operad are yet to be computed. As explained in the introduction, they should be given by the Ramanujan polynomials [3], which are polynomials in $\{x, y\}$ defined by

$$
\begin{aligned}
\psi_{1} & =1, \\
\psi_{n+1} & =\psi_{n}+(x+y)\left(n \psi_{n}+x \partial_{x} \psi_{n}\right) \quad n \geq 1 .
\end{aligned}
$$

More precisely, the dimension of the homogeneous component of degree $(i, j)$ of $\operatorname{Ram}(\{1,2, \ldots, n\})$ should be the coefficient of $x^{i} y^{j-i}$ in $\psi_{n}$.

This has been checked for sets with at most four elements. Besides, the parts of the bigraded dimensions corresponding to the Poisson and Bessel suboperads are correct, i.e. match the well-known dimensions of Poisson and the dimensions of Bessel computed in [2].

\subsection{Hopf structure}

In this section, a coproduct is defined which is compatible with composition, i.e. composition becomes a morphism of coalgebras.

The coproduct $\Delta$ is defined on generators by

$$
\left\{\begin{array}{l}
\Delta\left(E_{i, j}\right)=E_{i, j} \otimes E_{i, j}, \\
\Delta\left(L_{i, j}\right)=E_{i, j} \otimes L_{i, j}+L_{i, j} \otimes E_{i, j}, \\
\Delta\left(\Omega_{i, j}\right)=E_{i, j} \otimes \Omega_{i, j}+\Omega_{i, j} \otimes E_{i, j} .
\end{array}\right.
$$


Proposition 2 The coproduct $\Delta$ endows Ram with a structure of Hopf operad.

Proof One has to check the compatibility of $\Delta$ with all relations.

The cases of relations (1), (3) and (4) are well-know from the Hopf structure of the Poisson operad.

The case of relation (5) is a consequence of the study of the Bessel operad in $[2]$.

So there remains only to check the compatibility of the relation (2). Its coproduct is

$$
\begin{aligned}
& \sum_{c y c l}\left(E_{i, \star} \circ_{\star} E_{j, k}\right) \otimes\left(\Omega_{i, \star} \circ_{\star} L_{j, k}\right)+\left(E_{i, \star} \circ_{\star} L_{j, k}\right) \otimes\left(\Omega_{i, \star} \circ_{\star} E_{j, k}\right) \\
& +\left(\Omega_{i, \star} \circ_{\star} E_{j, k}\right) \otimes\left(E_{i, \star} \circ_{\star} L_{j, k}\right)+\left(\Omega_{i, \star} \circ_{\star} L_{j, k}\right) \otimes\left(E_{i, \star} \circ_{\star} E_{j, k}\right) \\
& +\left(L_{i, \star} \circ_{\star} \Omega_{j, k}\right) \otimes\left(E_{i, \star} \circ_{\star} E_{j, k}\right)+\left(L_{i, \star} \circ_{\star} E_{j, k}\right) \otimes\left(E_{i, \star} \circ_{\star} \Omega_{j, k}\right) \\
& \quad+\left(E_{i, \star} \circ_{\star} \Omega_{j, k}\right) \otimes\left(L_{i, \star} \circ_{\star} E_{j, k}\right)+\left(E_{i, \star} \circ_{\star} E_{j, k}\right) \otimes\left(L_{i, \star} \circ_{\star} \Omega_{j, k}\right) .
\end{aligned}
$$

By relations (3) and (2), this becomes

$$
\begin{aligned}
\sum_{c y c l}\left(E_{i, \star} \circ_{\star} L_{j, k}\right) \otimes\left(\Omega_{i, \star} \circ_{\star} E_{j, k}\right)+\left(\Omega_{i, \star} \circ_{\star} E_{j, k}\right) \otimes\left(E_{i, \star} \circ_{\star} L_{j, k}\right) \\
+\left(L_{i, \star} \circ_{\star} E_{j, k}\right) \otimes\left(E_{i, \star} \circ_{\star} \Omega_{j, k}\right)+\left(E_{i, \star} \circ_{\star} \Omega_{j, k}\right) \otimes\left(L_{i, \star} \circ_{\star} E_{j, k}\right) .
\end{aligned}
$$

By the distributive laws (4) and (5), this equals

$$
\begin{aligned}
& \sum_{c y c l}\left(E_{i, \star} \circ_{\star} L_{j, k}\right) \otimes\left(E_{j, \star} \circ_{\star} \Omega_{i, k}\right)+\left(E_{i, \star} \circ_{\star} L_{j, k}\right) \otimes\left(E_{k, \star} \circ_{\star} \Omega_{i, j}\right) \\
& +\left(E_{j, \star} \circ_{\star} \Omega_{i, k}\right) \otimes\left(E_{i, \star} \circ_{\star} L_{j, k}\right)+\left(E_{k, \star} \circ_{\star} \Omega_{i, j}\right) \otimes\left(E_{i, \star} \circ_{\star} L_{j, k}\right) \\
& +\left(E_{j, \star} \circ_{\star} L_{i, k}\right) \otimes\left(E_{i, \star} \circ_{\star} \Omega_{j, k}\right)+\left(E_{k, \star} \circ_{\star} L_{i, j}\right) \otimes\left(E_{i, \star} \circ_{\star} \Omega_{j, k}\right) \\
& \quad+\left(E_{i, \star} \circ_{\star} \Omega_{j, k}\right) \otimes\left(E_{j, \star} \circ_{\star} L_{i, k}\right)+\left(E_{i, \star} \circ_{\star} \Omega_{j, k}\right) \otimes\left(E_{k, \star} \circ_{\star} L_{i, j}\right) .
\end{aligned}
$$

Consider separately the terms of the form $(E \circ L) \otimes(E \circ \Omega)$ :

$$
\begin{aligned}
\sum_{c y c l}\left(E_{i, \star} \circ_{\star} L_{j, k}\right) \otimes\left(E_{j, \star} \circ_{\star} \Omega_{i, k}\right)+\left(E_{i, \star} \circ_{\star} L_{j, k}\right) \otimes\left(E_{k, \star} \circ_{\star} \Omega_{i, j}\right) \\
+\left(E_{j, \star} \circ_{\star} L_{i, k}\right) \otimes\left(E_{i, \star} \circ_{\star} \Omega_{j, k}\right)+\left(E_{k, \star} \circ_{\star} L_{i, j}\right) \otimes\left(E_{i, \star} \circ_{\star} \Omega_{j, k}\right) .
\end{aligned}
$$


Shifting the cyclic sum gives

$$
\begin{aligned}
\sum_{c y c l}\left(E_{i, \star} \circ_{\star}\right. & \left.L_{j, k}\right) \otimes\left(E_{j, \star} \circ_{\star} \Omega_{i, k}\right)+\left(E_{i, \star} \circ_{\star} L_{j, k}\right) \otimes\left(E_{k, \star} \circ_{\star} \Omega_{i, j}\right) \\
& +\left(E_{i, \star} \circ_{\star} L_{k, j}\right) \otimes\left(E_{k, \star} \circ_{\star} \Omega_{i, j}\right)+\left(E_{i, \star} \circ_{\star} L_{j, k}\right) \otimes\left(E_{j, \star} \circ_{\star} \Omega_{k, i}\right),
\end{aligned}
$$

which is zero. The same is true for terms of the form $(E \circ \Omega) \otimes(E \circ L)$. Therefore the coproduct of relation (2) is zero and the proposition is proved.

\subsection{Two Differentials}

Here are defined two differentials which are derivations for the composition and coderivations for the coproduct.

The differentials $D$ and $D^{\prime}$ are defined on generators by

$$
\left\{\begin{array} { l } 
{ D ^ { \prime } ( E _ { i , j } ) = 0 , } \\
{ D ^ { \prime } ( L _ { i , j } ) = \Omega _ { i , j } , } \\
{ D ^ { \prime } ( \Omega _ { i , j } ) = 0 }
\end{array} \quad \left\{\begin{array}{l}
D\left(E_{i, j}\right)=0 \\
D\left(\Omega_{i, j}\right)=L_{i, j} \\
D\left(L_{i, j}\right)=0
\end{array}\right.\right.
$$

Proposition 3 The differentials $D$ and $D^{\prime}$ can be extended to derivations for the composition.

Proof It is an easy exercise to check against all relations that the differentials can be extended to derivations.

Proposition 4 The differentials $D$ and $D^{\prime}$ are coderivations for the coproduct $\Delta$.

Proof This follows immediately by checking on generators using relations (9) and (10).

To summarize the results of this section, the Ram operad is a bigraded Hopf operad endowed with two differentials, which are derivations and coderivations, i.e. Ram is a Hopf operad in the chosen ambient category.

Algebraic 83 Geometric Topology, Volume 3 (2003) 


\section{The cooperad R}

\subsection{Abstract quotient algebras}

Let $I$ be a finite set. Consider the unital commutative associative algebra $\mathrm{R}(I)$ generated by elements $a_{i, j}$ antisymmetric of degree $(0,1)$ and $b_{i, j}$ antisymmetric of degree $(1,1)$ for all pairs of distinct elements $i, j$ of $I$ modulo the relations

$$
\begin{aligned}
a_{i, j}^{2} & =0, \\
a_{i, j} a_{j, k}+a_{j, k} a_{k, i}+a_{k, i} a_{i, j} & =0, \\
b_{i, j} a_{j, k}+b_{j, k} a_{k, i}+b_{k, i} a_{i, j}+a_{i, j} b_{j, k}+a_{j, k} b_{k, i}+a_{k, i} b_{i, j} & =0,
\end{aligned}
$$

the relations

$$
\begin{aligned}
a_{i_{0}, i_{1}} b_{i_{1}, i_{2}} b_{i_{2}, i_{3}} \ldots b_{i_{n}, i_{0}} & =0 \\
b_{i_{0}, i_{1}} b_{i_{1}, i_{2}} b_{i_{2}, i_{3}} \ldots b_{i_{n}, i_{0}} & =0
\end{aligned}
$$

for $n \geq 1$ where $i_{0}, i_{1}, i_{2}, \ldots, i_{n}$ are pairwise different elements of $I$, and the 12 -terms relations

$$
\begin{aligned}
& b a b_{i, j, k, \ell}+b a b_{i, k, j, \ell}+b a b_{i, j, \ell, k}+b a b_{i, \ell, j, k}+b a b_{i, k, \ell, j}+b a b_{i, \ell, k, j} \\
& \quad+b a b_{j, i, k, \ell}+b a b_{j, k, i, \ell}+b a b_{j, i, \ell, k}+b a b_{j, \ell, i, k}+b a b_{k, i, j, \ell}+b a b_{k, j, i, \ell}=0, \\
& \quad b b b_{i, j, k, \ell}+b b b_{i, k, j, \ell}+b b b_{i, j, \ell, k}+b b b_{i, \ell, j, k}+b b b_{i, k, \ell, j}+b b b_{i, \ell, k, j} \\
& \quad+b b b_{j, i, k, \ell}+b b b_{j, k, i, \ell}+b b b_{j, i, \ell, k}+b b b_{j, \ell, i, k}+b b b_{k, i, j, \ell}+b b b_{k, j, i, \ell}=0,
\end{aligned}
$$

where $b a b_{i, j, k, \ell}=b_{i, j} a_{j, k} b_{k, \ell}$ and $b b b_{i, j, k, \ell}=b_{i, j} b_{j, k} b_{k, \ell}$ for short.

Remark that the 12 terms in relations (16) and (17) correspond to permutations up to reversal.

Note that the subalgebra of elements of first degree 0 (i.e. generated by the elements $a_{i, j}$ ) has already appeared in the work of Mathieu on the symplectic and Poisson operads [9] (see also [6, §4.3] and [10]).

Lemma 1 One has

$$
\square_{i_{0}, i_{1}} \square_{i_{1}, i_{2}} \square_{i_{2}, i_{3}} \ldots \square_{i_{n}, i_{0}}=0,
$$

for $n \geq 1$ where $i_{0}, i_{1}, i_{2}, \ldots, i_{n}$ are pairwise different elements of $I$ and the empty boxes are filled by $a$ and $b$ in an arbitrary way.

Algebraic 83 Geometric Topology, Volume 3 (2003) 
Proof If there is no $a$ at all, equation (18) is just equation (15). If there is exactly one $a$, then one can use commutativity to assume without further restrictions that this $a$ is the leftmost letter, which gives equation (14). Therefore one can assume from now on that there are at least two $a$.

The proof is by recursion on the length $n$ of the cycle. If $n=1$, then the statement is true by relation (11). Assume that $n \geq 2$ and the statement is true for all integers less than $n$.

The proof is now by another recursion on the shortest chain of $b$ between two $a$.

Assume first that there are two adjacent $a$ in the cycle, say $a_{i_{0}, i_{1}} a_{i_{1}, i_{2}}$. Then one can use relation (12) to replace $a_{i_{0}, i_{1}} a_{i_{1}, i_{2}}$ by a sum of two terms in the product. Each of the two products obtained contains a shorter cycle and therefore vanish.

Assume that there are no adjacent $a$ in the cycle. Consider the shortest chain of $b$ between two $a$. One can assume without restriction that one of these $a$ and one $b$ in the shortest chain are $a_{i_{0}, i_{1}} b_{i_{1}, i_{2}}$. By using relation (13), one can replace $a_{i_{0}, i_{1}} b_{i_{1}, i_{2}}$ by a sum of five terms in the product. Among the five products obtained, four have a shorter cycle and one has a shorter chain of $b$ between two $a$. Therefore all these products vanish by recursion.

The recursion on the chain is done. The recursion on $n$ is done.

Lemma 2 The algebras $\mathrm{R}(I)$ are finite-dimensional. The second grading takes values between 0 and the cardinality of I minus one.

Proof One can map each monomial to a graph on the set $I$ with edges colored by $a$ and $b$. If this graph has multiple edges, the monomial vanishes by relation (11) and relations (14) and (15) for $n=1$. If this graph has a loop, the corresponding monomial vanishes by Lemma 1 . Therefore only monomials corresponding to forests of simple trees can be non-zero in $\mathrm{R}(I)$. In such a forest, the number of edges is at most one less than the cardinality of $I$. As the generators have second degree 1, the maximal second degree of a non-zero monomial is therefore bounded by the cardinality of $I$ minus one.

Lemma 3 One has

$$
\sum_{\sigma}(a a b)_{\sigma}=0,
$$

where $\sigma$ runs over the set of permutations of $\{i, j, k, \ell\}$ and

$$
(a a b)_{\sigma}=a_{\sigma(i), \sigma(j)} a_{\sigma(j), \sigma(k)} b_{\sigma(k), \sigma(\ell)} .
$$


One has

$$
\sum_{\left(i^{\prime}, i^{\prime \prime}\right)} T_{i^{\prime}}^{i^{\prime \prime}}=0
$$

where

$$
T_{i}^{j}=b_{i, j} a_{i, k} a_{i, \ell}
$$

and the sum is over pairs of distinct elements of $\{i, j, k, \ell\}$.

Proof Let us call $\Sigma$ the first sum and $T$ the second sum.

Both statements are proved simultaneously. Consider the simplex with vertex set $\{i, j, k, \ell\}$. To a facet $f$, one can associate a relation $r(f)$ of type (13) and a relation $s(f)$ of type (12). To an edge $e$, one can associate an element $a(e)$ and an element $b(e)$.

By summing (with appropriate signs) $r(f) a(e)$ over the set of pairs $(f, e)$ where $f$ is a facet and $e$ an edge such that $e \not \subset f$, one gets that $\Sigma+2 T$ vanishes.

By summing (with appropriate signs) $s(f) b(e)$ over the set of pairs $(f, e)$ where $f$ is a facet and $e$ an edge such that $e \not \subset f$, one gets that $\Sigma+T$ vanishes.

Lemma 4 One has

$$
\sum_{\sigma}(a b b)_{\sigma}=0
$$

where $\sigma$ runs over the set of permutations of $\{i, j, k, \ell\}$ and

$$
(a b b)_{\sigma}=a_{\sigma(i), \sigma(j)} b_{\sigma(j), \sigma(k)} b_{\sigma(k), \sigma(\ell)} .
$$

Proof Consider the simplex with vertex set $\{i, j, k, \ell\}$. To a facet $f$, one can associate a relation $r(f)$ of type (13). To an edge $e$, one can associate an element $b(e)$.

By summing (with appropriate signs) $r(f) b(e)$ over the set of pairs $(f, e)$ where $f$ is a facet and $e$ an edge such that $e \not \subset f$, one gets that the sum (21) vanishes.

One can define two differentials $d$ and $d^{\prime}$ on generators by

$$
\left\{\begin{array} { l } 
{ d ( a _ { i , j } ) = b _ { i , j } , } \\
{ d ( b _ { i , j } ) = 0 . }
\end{array} \quad \left\{\begin{array}{l}
d^{\prime}\left(b_{i, j}\right)=a_{i, j}, \\
d^{\prime}\left(a_{i, j}\right)=0 .
\end{array}\right.\right.
$$


Proposition 5 The differentials $d$ and $d^{\prime}$ can be extended to derivations of the algebra $\mathrm{R}(I)$.

Proof The check is quite easy for the differential $d$. For $d^{\prime}$, the only non-trivial cases are relations (14), (16) and (17).

The case of relation (14) is settled by Lemma 1 .

The image by $d^{\prime}$ of relation (16) is exactly the sum (19) which vanishes by Lemma 3.

The image by $d^{\prime}$ of relation (17) is the sum of relation (16) and relation (21) and therefore vanishes by Lemma 4 .

\subsection{Cocomposition maps}

Let $I$ and $J$ be two finite sets. Motivated by the similar cocomposition (40) for the dual of the Gerstenhaber operad, one defines the cocomposition map $\Theta_{I, J}^{\star}$ from $I \sqcup J$ to $(I \sqcup\{\star\}, J)$ on generators by

$$
\Theta_{I, J}^{\star}\left(a_{i, j}\right)=\left\{\begin{array}{l}
a_{i, j} \otimes 1 \text { if } i, j \in I, \\
1 \otimes a_{i, j} \text { if } i, j \in J, \\
a_{i, \star} \otimes 1 \text { if } i \in I \text { and } j \in J
\end{array}\right.
$$

and

$$
\Theta_{I, J}^{\star}\left(b_{i, j}\right)=\left\{\begin{array}{l}
b_{i, j} \otimes 1 \text { if } i, j \in I, \\
1 \otimes b_{i, j} \text { if } i, j \in J, \\
b_{i, \star} \otimes 1 \text { if } i \in I \text { and } j \in J
\end{array}\right.
$$

Proposition 6 This defines morphisms $\Theta_{I, J}^{\star}$ of bidifferential algebras from $\mathrm{R}(I \sqcup J)$ to $\mathrm{R}(I \sqcup\{\star\}) \otimes \mathrm{R}(J)$.

Proof First one has to check against all relations that $\Theta_{I, J}^{\star}$ can be extended to a morphism of algebras. By the very simple shape of cocomposition, the compatibility is clear if all indices involved are in $J$ or if all but maybe one are in $I$.

So one can assume that there is at least one index in $I$ and at least two indices in $J$. Again compatibility is easy to check for all relations involving at most three indices. The only non-trivial cases are the relations (14) and (15) and the 12 -terms relations (16) and (17). 
Consider first the case of relations (14) and (15). More generally, consider any cycle $\square_{i_{0}, i_{1}} \square_{i_{1}, i_{2}} \ldots \square_{i_{n}, i_{0}}$, where boxes are either $a$ or $b$.

As there is at least one index of the cycle in $I$ and at least two in $J$, one can assume without restriction that $i_{0} \in J, i_{1}, \ldots, i_{k} \in I$ and $i_{k+1} \in J$ for some $k \geq 1$. Then the left tensor in the image by $\Theta_{I, J}^{\star}$ of the cycle contains the cycle $\square_{\star, i_{1}} \square_{i_{1}, i_{2}} \ldots \square_{i_{k}, \star}$ for some $a$ and $b$ in the boxes, and therefore vanishes by Lemma 1 .

Now consider for example the case of (16) with $i, j \in I$ and $k, \ell \in J$. Its cocomposition is given by

$$
\begin{aligned}
\left(b_{i, j} \otimes 1\right) & \left(a_{j, \star} \otimes 1\right)\left(1 \otimes b_{k, \ell}\right)+\left(b_{i, \star} \otimes 1\right)\left(a_{\star, j} \otimes 1\right)\left(b_{j, \star} \otimes 1\right) \\
+ & \left(b_{i, j} \otimes 1\right)\left(a_{j, \star} \otimes 1\right)\left(1 \otimes b_{\ell, k}\right)+\left(b_{i, \star} \otimes 1\right)\left(a_{\star, j} \otimes 1\right)\left(b_{j, \star} \otimes 1\right) \\
+ & \left(b_{i, \star} \otimes 1\right)\left(1 \otimes a_{k, \ell}\right)\left(b_{\star, j} \otimes 1\right)+\left(b_{i, \star} \otimes 1\right)\left(1 \otimes a_{\ell, k}\right)\left(b_{\star, j} \otimes 1\right) \\
+ & \left(b_{j, i} \otimes 1\right)\left(a_{i, \star} \otimes 1\right)\left(1 \otimes b_{k, \ell}\right)+\left(b_{j, \star} \otimes 1\right)\left(a_{\star, i} \otimes 1\right)\left(b_{i, \star} \otimes 1\right) \\
+ & \left(b_{j, i} \otimes 1\right)\left(a_{i, \star} \otimes 1\right)\left(1 \otimes b_{\ell, k}\right)+\left(b_{j, \star} \otimes 1\right)\left(a_{\star, i} \otimes 1\right)\left(b_{i, \star} \otimes 1\right) \\
& +\left(b_{\star, i} \otimes 1\right)\left(a_{i, j} \otimes 1\right)\left(b_{j, \star} \otimes 1\right)+\left(b_{\star, j} \otimes 1\right)\left(a_{j, i} \otimes 1\right)\left(b_{i, \star} \otimes 1\right) .
\end{aligned}
$$

This is equal to

$$
\begin{gathered}
\left(b_{i, j} a_{j, \star}\right) \otimes b_{k, \ell}+\left(b_{i, \star} a_{\star, j} b_{j, \star}\right) \otimes 1+\left(b_{i, j} a_{j, \star}\right) \otimes b_{\ell, k}+\left(b_{i, \star} a_{\star, j} b_{j, \star}\right) \otimes 1 \\
+\left(b_{i, \star} b_{\star, j}\right) \otimes a_{k, \ell}+\left(b_{i, \star} b_{\star, j}\right) \otimes a_{\ell, k}+\left(b_{j, i} a_{i, \star}\right) \otimes b_{k, \ell}+\left(b_{j, \star} a_{\star, i} b_{i, \star}\right) \otimes 1 \\
+\left(b_{j, i} a_{i, \star}\right) \otimes b_{\ell, k}+\left(b_{j, \star} a_{\star, i} b_{i, \star}\right) \otimes 1+\left(b_{\star, i} a_{i, j} b_{j, \star}\right) \otimes 1+\left(b_{\star, j} a_{j, i} b_{i, \star}\right) \otimes 1 .
\end{gathered}
$$

Using antisymmetry and some relations, this is seen to be zero. The proof in the remaining cases for (16) and (17) is similar and left to the reader.

This map clearly respects both differentials, as can be checked on generators.

Proposition 7 The applications $\Theta$ define a cooperad structure on R.

Proof One has to check on the generators of $\mathrm{R}(I \sqcup J \sqcup K)$ that

$$
\left(\Theta_{I, J \sqcup\{\#\}}^{\star} \otimes I d_{K}\right) \circ \Theta_{I \sqcup J, K}^{\#}=\left(I d_{I \sqcup\{\star\}} \otimes \Theta_{J, K}^{\#}\right) \circ \Theta_{I, J \sqcup K}^{\star}
$$

and that

$$
\left(\Theta_{I \sqcup\{\#\}, J}^{\star} \otimes I d_{K}\right) \circ \Theta_{I \sqcup J, K}^{\#}=\left(I d_{I} \otimes \tau\right) \circ\left(\Theta_{I \sqcup\{\star\}, K}^{\#} \otimes I d_{J}\right) \circ \Theta_{I \sqcup K, J}^{\star},
$$

where $\tau$ is the symmetry isomorphism for $\mathrm{R}(J) \otimes \mathrm{R}(K)$. 
The proof is case by case according to the indices of the generator. Consider for example equation (27) and a generator $a_{i, j}$ in $\mathrm{R}(I \sqcup J \sqcup K)$ with $i \in I$ and $j \in K$. One has on the one hand

$$
\left(\Theta_{I, J \sqcup\{\#\}}^{\star} \otimes I d_{K}\right) \circ \Theta_{I \sqcup J, K}^{\#}\left(a_{i, j}\right)=\left(\Theta_{I, J \sqcup\{\#\}}^{\star} \otimes I d_{K}\right)\left(a_{i, \#} \otimes 1\right)=a_{i, \star} \otimes 1 \otimes 1 .
$$

On the other hand,

$$
\left(I d_{I \sqcup\{\star\}} \otimes \Theta_{J, K}^{\#}\right) \circ \Theta_{I, J \sqcup K}^{\star}\left(a_{i, j}\right)=\left(I d_{I \sqcup\{\star\}} \otimes \Theta_{J, K}^{\#}\right)\left(a_{i, \star} \otimes 1\right)=a_{i, \star} \otimes 1 \otimes 1 .
$$

The remaining cases are similar and left to the reader.

\subsection{Morphism of operads}

Here is defined a morphism $\rho$ from the operad Ram to the dual operad $\mathrm{R}^{*}$ of the cooperad R.

Consider the dual vector space $\mathrm{R}^{*}(I)$ of $\mathrm{R}(I)$. This vector space is bigraded. Define elements $1^{*}, b_{i, j}^{*}, a_{i, j}^{*}$ in $\mathrm{R}^{*}(I)$ as the dual basis (with respect to the pairing $\left.\mathrm{R}(I) \otimes \mathrm{R}^{*}(I) \rightarrow \mathbb{C}\right)$ for the components of degree $(0,0),(0,1)$ and $(1,1)$ respectively.

The map $\rho$ is defined on the generators of Ram by

$$
\left\{\begin{array}{l}
E_{i, j} \mapsto 1^{*}, \\
\Omega_{i, j} \mapsto b_{i, j}^{*}, \\
L_{i, j} \mapsto a_{i, j}^{*}
\end{array}\right.
$$

Proposition 8 This defines a map $\rho$ of Hopf operads from Ram to $\mathrm{R}^{*}$. The map $\rho$ intertwines $d$ with $D^{*}$ and $d^{\prime}$ with $\left(D^{\prime}\right)^{*}$.

Proof First, one has to check that this indeed defines a morphism of operads, i.e. the compatibility with relations defining Ram.

For example, let us check the compatibility for relation (2). By the bigrading, it is sufficient to prove that the corresponding linear form vanishes on the dual bihomogeneous component. First compute the following cocompositions:

$$
\begin{aligned}
& \Theta_{\{i\},\{j, k\}}^{\star}\left(a_{i, j} b_{j, k}\right)=a_{i, \star} \otimes b_{j, k}, \\
& \Theta_{\{i\},\{j, k\}}^{\star}\left(a_{j, k} b_{k, i}\right)=b_{\star, i} \otimes a_{j, k}, \\
& \Theta_{\{i\},\{j, k\}}^{\star}\left(a_{k, i} b_{i, j}\right)=0, \\
& \Theta_{\{i\},\{j, k\}}^{\star}\left(b_{i, j} a_{j, k}\right)=b_{i, \star} \otimes a_{j, k}, \\
& \Theta_{\{i\},\{j, k\}}^{\star}\left(b_{j, k} a_{k, i}\right)=a_{\star, i} \otimes b_{j, k}, \\
& \Theta_{\{i\},\{j, k\}}^{\star}\left(b_{k, i} a_{i, j}\right)=0 .
\end{aligned}
$$


From this, one can deduce a description of the linear forms $a_{i, \star}^{*} \circ_{\star} b_{j, k}^{*}$ and $b_{i, \star}^{*} \circ_{\star} a_{j, k}^{*}$ by their values on a basis of the homogeneous component of degree $(1,2)$ of $\mathrm{R}(\{i, j, k\})$.

Now the sum (2) is mapped by $\rho$ to

$$
\sum_{c y c l}\left(b_{i, \star}^{*} \circ_{\star} a_{j, k}^{*}+a_{i, \star}^{*} \circ_{\star} b_{j, k}^{*}\right) .
$$

One then checks that this sum vanishes as a linear form.

The proof of compatibility for the other relations is similar.

The intertwining property for differentials is clear on the generators $\operatorname{Ram}(\{i, j\})$ of Ram. It is also easy to prove that this map is a morphism of coalgebras by checking on generators of Ram.

That the map $\rho$ is an isomorphism has been checked for sets with at most three elements. Furthermore the bigraded dimensions of Ram and $\mathrm{R}^{*}$ coincide for sets with at most four elements. One can therefore ask the following

Question Is $\rho$ an isomorphism?

Remark that it follows from the fact that $\rho$ is a morphism of Hopf operads that, by transposition, the relations of the algebras underlying $\mathrm{R}$ are satisfied in the dual algebras of the coalgebras underlying Ram.

\subsection{Algebras of differential forms}

Here, a tentative relation of $\mathrm{R}$ with differential forms on hyperplane arrangements is proposed.

Let $I$ be a finite set and $\mathbb{C}^{I}$ be the vector space with coordinates $\left(x_{i}\right)_{i \in I}$. Let $\mathrm{H}_{I}$ be the union of all hyperplanes $x_{i}-x_{j}=0$ for $i \neq j$ in the subspace $\sum_{i \in I} x_{i}=0$ of $\mathbb{C}^{I}$ (this is a type $A$ hyperplane arrangement). Consider the subalgebra of the algebra of differential forms with poles along $\mathrm{H}_{I}$ generated over $\mathbb{C}$ by elements $a_{i, j}=1 /\left(x_{i}-x_{j}\right)$ and $b_{i, j}=d\left(1 /\left(x_{i}-x_{j}\right)\right)$ for $i \neq j$ (here $d$ is the de Rham differential).

The elements $a_{i, j}$ and $b_{i, j}$ are antisymmetric. There are two natural gradings on this algebra: the first one is by the degree as a differential form, the second one is the homogeneity degree where all variables $x_{i}$ are taken homogeneous of degree minus one. 
One has the following relations:

$$
\begin{aligned}
a_{i, j} a_{j, k}+a_{j, k} a_{k, i}+a_{k, i} a_{i, j} & =0, \\
b_{i, j} a_{j, k}+b_{j, k} a_{k, i}+b_{k, i} a_{i, j}+a_{i, j} b_{j, k}+a_{j, k} b_{k, i}+a_{k, i} b_{i, j} & =0,
\end{aligned}
$$

and

$$
b_{i_{0}, i_{1}} b_{i_{1}, i_{2}} b_{i_{2}, i_{3}} \ldots b_{i_{n}, i_{0}}=0
$$

for $n \geq 1$ where $i_{0}, i_{1}, i_{2}, \ldots, i_{n}$ are pairwise different elements of $I$.

It is plausible that the abstract algebras $\mathrm{R}(I)$ introduced before are quotients of these concrete algebras of differential forms. The main problem is to find some geometric reason for the relations of $\mathrm{R}(I)$.

\subsection{On the Gerstenhaber operad}

This section is mainly for motivation and details are therefore omitted.

Recall the topological little discs operad $\mathrm{D}^{2}$, where $\mathrm{D}^{2}(I)$ is the space of disjoint embeddings of scaled unit discs, bijectively labeled by $I$, inside a unit disc. The composition inside a little disc is obtained by replacing this little disc by a collection of little discs appropriately scaled, see [11] for further details.

The algebra $\mathcal{O}_{\mathrm{D}^{2}}(I)$ defined by

$$
\mathbb{C}\left[x_{i}\right]\left[\left(x_{i}-x_{j}\right)^{-1}\right]\left[\left[\epsilon_{i}\right]\right]\left[\epsilon_{i}^{-1}\right]
$$

is an algebraic analog of the algebra of functions on the space $\mathrm{D}^{2}(I)$, where the $x$ variables are the pairwise-different complex coordinates of the centers and the $\epsilon$ variables are the infinitesimal non-vanishing real radiuses. Assuming that radiuses are infinitesimal ensures disjointness of discs. One can easily translate the composition rule of the topological little discs operad into a cocomposition rule defining a cooperad on the collection of the algebras $\mathcal{O}_{\mathrm{D}^{2}}(I)$ for all finite sets $I$.

Now the Gerstenhaber operad can be defined as the homology of the little discs operad $[7,4,5]$. As the space $\mathrm{D}^{2}(I)$ is homotopy equivalent to the complement of a complexified hyperplane arrangement of type $A$, a theorem of Arnold [1] implies that its cohomology is generated by the classes of the differential forms

$$
\omega_{i, j}=d\left(\log \left(x_{i}-x_{j}\right)\right),
$$

subject only to the relations

$$
\omega_{i j} \omega_{j k}+\omega_{j k} \omega_{k i}+\omega_{k i} \omega_{i j}=0
$$


One can extend the algebraic cocomposition rules for the collection of algebras $\mathcal{O}_{\mathrm{D}^{2}}(I)$ obtained before to cocomposition rules for the collection of algebras of differential forms of $\mathcal{O}_{\mathrm{D}^{2}}(I)$ with respect to the $x$ variables. It is then possible to restrict these rules to the collection of subalgebras generated by the forms $\omega_{i, j}$. The result is as follows for the cocomposition map $\Theta_{I, J}^{\star}$ from $I \sqcup J$ to $(I \sqcup\{\star\}, J):$

$$
\Theta_{I, J}^{\star}\left(\omega_{i, j}\right)=\left\{\begin{array}{l}
\omega_{i, j} \otimes 1 \text { if } i, j \in I, \\
1 \otimes \omega_{i, j} \text { if } i, j \in J, \\
\omega_{i, \star} \otimes 1 \text { if } i \in I \text { and } j \in J .
\end{array}\right.
$$

Together with the Arnold relations (39), this provides an algebraic description of the dual cooperad of the Gerstenhaber operad.

\section{References}

[1] V I Arnold, The cohomology ring of the group of dyed braids, Mat. Zametki 5 (1969) 227-231

[2] F Chapoton, A Hopf operad of forests of binary trees and related finitedimensional algebras. (Sept. 2002), arXiv:math.CO/0209038

[3] W Y C Chen, V J W Guo, Bijections behind the Ramanujan polynomials, Adv. in Appl. Math. 27 (2001) 336-356, special issue in honor of Dominique Foata's 65th birthday (Philadelphia, PA, 2000)

[4] F R Cohen, The homology of $C_{n+1}$-spaces, $n \geq 0$, from: "The homology of iterated loop spaces.", Lecture Notes in Mathematics 533, Springer (1976)

[5] F R Cohen, Artin's braid groups, classical homotopy theory, and sundry other curiosities, from: "Braids (Santa Cruz, CA, 1986)", Contemp. Math. 78, Amer. Math. Soc., Providence, RI (1988) 167-206

[6] S Fomin, A N Kirillov, Quadratic algebras, Dunkl elements, and Schubert calculus, from: "Advances in geometry", Progr. Math. 172, Birkhäuser Boston, Boston, MA (1999) 147-182

[7] M Gerstenhaber, A A Voronov, Homotopy G-algebras and moduli space operad, Internat. Math. Res. Notices (1995) 141-153 (electronic)

[8] M Markl, Distributive laws and Koszulness, Ann. Inst. Fourier (Grenoble) 46 (1996) 307-323

[9] O Mathieu, The symplectic operad, from: "Functional analysis on the eve of the 21st century, Vol. 1 (New Brunswick, NJ, 1993)", Progr. Math. 131, Birkhäuser Boston, Boston, MA (1995) 223-243

[10] A Varchenko, I Gelfand, Heaviside functions of a configuration of hyperplanes, Funktsional. Anal. i Prilozhen. 21 (1987) 1-18, 96 
[11] A A Voronov, The Swiss-cheese operad, from: "Homotopy invariant algebraic structures (Baltimore, MD, 1998)", Contemp. Math. 239, Amer. Math. Soc., Providence, RI (1999) 365-373

\section{LACIM}

Université du Québec à Montréal

CP 8888 succ. centre ville

Montréal Québec H3C 3P8

Canada

Email: chapoton@math.uqam.ca

Received: 20 January 2003 Revised: 12 December 2003 